\title{
Impact of temperature on short- and long-term aging of asphalt binders
}

\author{
Lily D. Poulikakos ${ }^{\mathrm{a} *}$, Bernhard Hofko $^{\mathrm{b}}$, Laurent Porot ${ }^{\mathrm{c}}$, Xiaohu Lu $^{\mathrm{d}}$, Hartmut Fischer ${ }^{\mathrm{e}}$, Nicole Kringos ${ }^{\mathrm{f}}$
}

\author{
${ }^{a}$ Empa, Swiss Federal Laboratories for Materials Science and Technology, Switzerland \\ ${ }^{\mathrm{b}}$ Vienna University of Technology, Austria \\ ${ }^{c}$ Arizona Chemical, The Netherlands \\ ${ }^{d}$ Nynas AB, Sweden \\ e TNO Technical Sciences, The Netherlands \\ ${ }^{\mathrm{f}} \mathrm{KTH}$, Royal Institute of Technology, Sweden
}

Received: 13 November 2015 / Accepted: 15 January 2016 / Published online: 17 March 2016

(C) The Author(s) 2016. This article is published with open access.

\begin{abstract}
Properties of asphalt concrete after aging are important parameters in determining the long-term performance of these materials. With the popularity of reduced temperature mixtures the question remains how this reduced temperature in short-term aging affects the long-term properties. This paper focuses on developing a robust and fundamental understanding of the effect of temperature on aging by connecting the chemistry of bitumen to its mechanics. To this end, round robin experiments are being currently conducted within 8 laboratories using four binders of the same grade $70 / 100$ pen from different crude sources. In developing chemo-mechanical characterization techniques at the nano- and micro-scale, the material's variability from crude-source to crude-source and its sensitivity to temperature needs to be taken into consideration. Furthermore, the development of uniform specimen preparation procedures for these bituminous materials at the nano- and micro-structural level is the focus. The chemical characterization is performed using Fourier transform infrared spectroscopy (FTIR). For the mechanical characterization rheological data is used using the dynamic shear rheometer (DSR) as well as conventional tests, e.g. needle penetration and softening point using the ring and ball method. It is shown that the shortterm aging temperature affects the increase in softening point, while the consequent effect on long term aging is less pronounced.
\end{abstract}

Keywords: Bitumen; Aging; Rheology; FTIR; Microstructure

\section{Introduction}

Bituminous materials can have very different long-term properties, depending on the source they come from and production refining process. Yet most of the research towards enhanced properties of bituminous materials is largely split into separate efforts towards understanding its chemistry or its mechanics. Connecting these two research fields into a joined chemo-mechanical understanding could lead to major improvements in the material.

Since the long-term response of asphalt mixtures under varying environmental (i.e. moisture, UV and oxygen) and mechanical (i.e. traffic) loading is crucial, having a fundamental understanding of the combined chemomechanical properties can greatly enhance the material's sustainability and functionality. Thus, in developing chemomechanical characterization techniques at the nano- and micro-scale, the material's variability from crude-source to crude-source and its sensitivity to temperature and aging need to be taken into consideration.
The goals of this cooperative research effort are:

i. Identifying chemo-mechanically coupled properties of selected bituminous materials (literature study, state of the art, STAR);

ii. Quantifying dominant parameters of the selected chemo-mechanical properties by following uniform experimental testing procedures (round robin tests);

iii. Developing and testing the influence of specimen preparation techniques (round robin tests);

iv. Recommending appropriate tools and techniques for the chemo-mechanical characterization (recommendations).

The identification of the chemo-mechanically coupled properties that are investigated was based on a literature survey of the existing knowledge in the bituminous materials field as well as other building and non-building materials as outlined in (i) above. At a first stage, properties were selected and the appropriate chemo-mechanical experimental procedures were chosen. To this end, specimen preparation and test protocols were refined as outlined in (ii) and (iii) above. This will lead to detailed recommendations for improved and potentially new standards as outlined in (iv) above. 
In order to achieve these goals a series of round robin tests is currently in progress and the preliminary results are presented here. The round robin tests aim to analyze the impact of temperature on accelerated laboratory aging simulating short- and long-term aging (referred to as short and long term aging hereafter) in the lab for one type of asphalt binder (70/100 pen) from different crude sources. The motivation for this work is the technology for temperature-reduced mixtures (warm-mix asphalt WMA), which has gained importance in recent years. There is an on-going scientific debate on whether temperature reduction for asphalt mixture production, which leads to reduced short-term aging, brings additional benefits in terms of reducing long-term aging.

\section{Microstructural considerations}

Research done by the authors has shown that there is a change in the microstructure with increasing temperatures $[1,2]$. Furthermore, different phases vary in physical state (at different T) and different chemical compositions (Figure 1). This means that those parts of the binder which are forming the solid elastic phase will age differently than other parts forming the viscous continuous phase. However, almost all artificial aging techniques use elevated temperatures $\left(\sim 60^{\circ} \mathrm{C}\right)$ above which microstructure disappears. By aging thin films of binder in an oven at $163^{\circ} \mathrm{C}$, the rolling thin film oven test (RTFOT) simulates short-term aging in the laboratory that represents aging before the material is placed in the road [3]. The pressure aging vessel (PAV) uses temperature of $\sim 90-110{ }^{\circ} \mathrm{C}$ and a pressure $2.10 \mathrm{MPa}$ to simulate long-term aging in the laboratory [4]. Thus, the material in the artificial aging state is completely different from that at a temperature below $\sim 80{ }^{\circ} \mathrm{C}$ (e.g. roads). Consequently, in developing future standardized aging tests, it should be noted that useful conclusions from aging experiments with respect to performance may only be possible while performing such experiments in a temperature range where still microstructures are present. In addition, the RTFOT test should be used to study aging during mixing with fillers and aggregates, as these components also have a significant effect on the short-term aging. However this also adds an extra level of complexity to an already complex phenomena.

\section{Outline}

The round robin tests currently in progress compare changes in mechanical and chemical characteristics of samples due to RTFOT aging at 3 different temperatures as well as changes due to subsequent standard PAV aging. The mechanical analysis comprises standard tests: penetration [5], softening point ring \& ball [6] and dynamic shear rheometer (DSR) testing. Chemical analysis is carried out using attenuated total reflectance fourier transform infrared spectroscopy (ATR-FTIR). Each lab runs the test program for at least the standard RTFOT temperature, $163{ }^{\circ} \mathrm{C}$ and one additional temperature selected between $123{ }^{\circ} \mathrm{C}$ and $143{ }^{\circ} \mathrm{C}$, to keep the work load within reasonable limits. In parallel, asphalt mixtures will be produced with one of the employed binders at one participant lab at a reduced temperature by using foamed binder and at another participant lab at standard temperatures with the virgin binder. The binder will be extracted from both mixtures and compared to the results of lab-aged binders using RTFOT at different temperatures. Furthermore, there will be a comparison of RTFOT and PAV with field aging. The experimental program for the binder phase is shown in Figure 2.

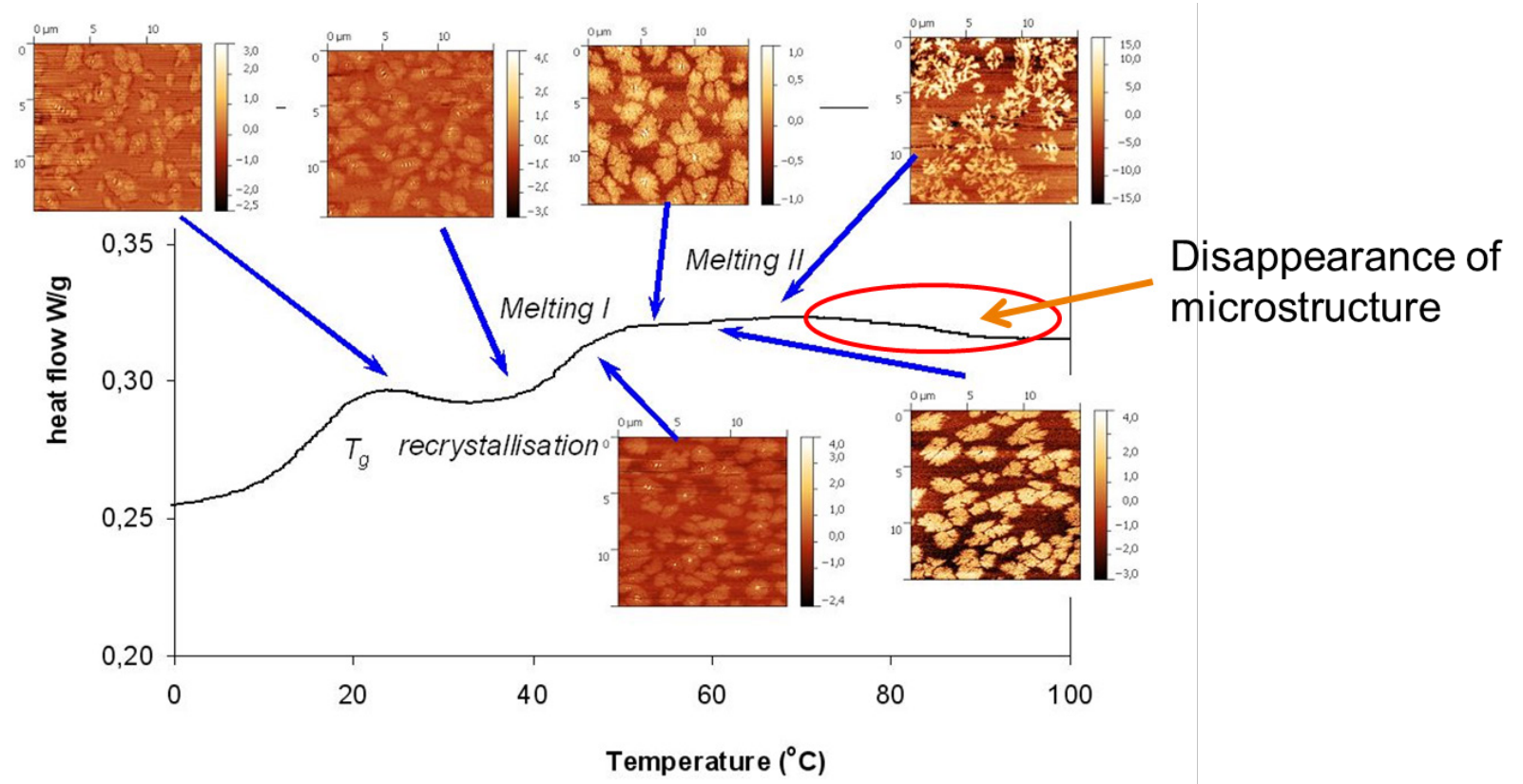

Figure 1. Microstructural changes with increasing temperature [1]. 


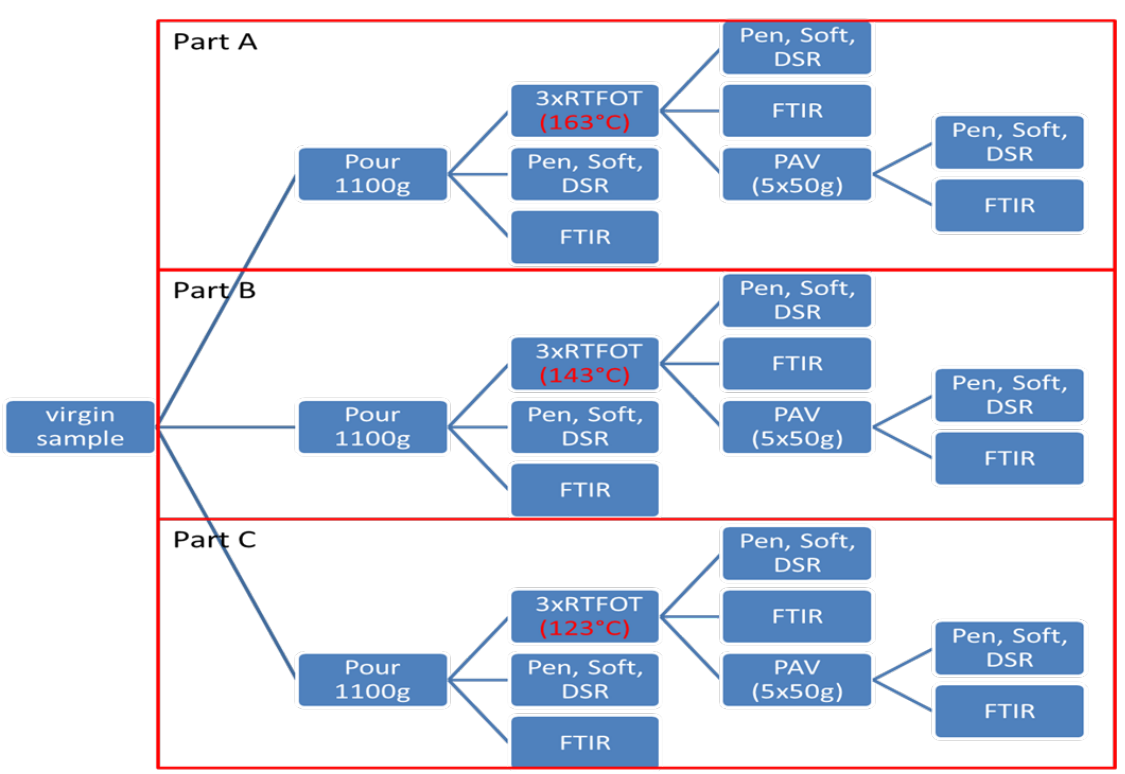

Figure 2. Experimental program of the round robin tests for binder evaluation.

\section{Materials}

Four samples of 70/100 pen-graded binders were provided from four suppliers. Each participant received $2.5 \mathrm{~kg}$ of binder sample. This allows comparison of relevant characteristics of bitumen from different crude sources.

\section{Preliminary results}

Figure 3 shows preliminary results of the complex modulus at different temperatures for one of the tested binders. It can be seen that the complex modulus of the RTFOT-PAV aged sample is considerably higher at all tested temperatures indicating aging and stiffening of the RTFOT at $163{ }^{\circ} \mathrm{C}$ and PAV aged binder. Furthermore the complex modulus of the virgin binder is the lowest followed by the RTFOT at $123{ }^{\circ} \mathrm{C}$ and $163{ }^{\circ} \mathrm{C}$. This indicates that the lower temperature of the short-term aging procedure has a direct effect on the rheological properties of the binder. What remains to be seen is if this also manifests itself into reduced aging after PAV.

\section{DSR Results - 70/100 at different aging levels}

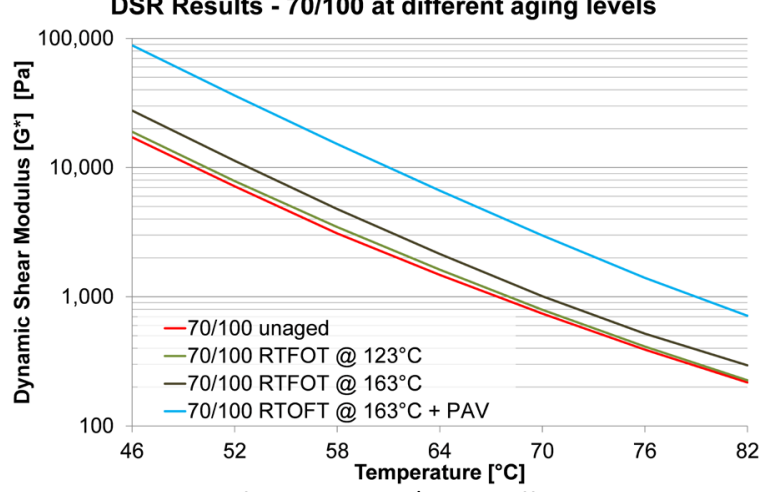

Additional testing, run on a single bituminous binder with 4 different RTFOT temperatures from $123{ }^{\circ} \mathrm{C}$ till $183{ }^{\circ} \mathrm{C}$ followed by PAV, can show the relative changes of these properties. As an example, Figure 4 shows the change in softening point vs. temperature, indicating that the RTFOT temperature affects the increase in softening point, while after PAV this difference is less pronounced.

\section{Softening point B505 over RTFOT Temperature}

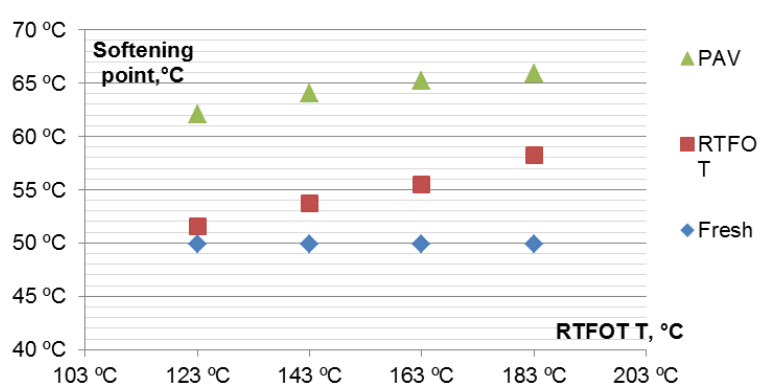

Figure 4. Softening point change with RTFOT temperature.

In order to characterize chemical changes in the binders as a result of oxidation due to artificial aging, ATR-FTIR was used. ATR-FTIR measurements allow the identification of certain functional groups in bitumen. From this measurement Carbonyl and Sulfoxide Indexes are determined by the area method, respectively between $1660-1753 \mathrm{~cm}^{-1}$ and $1047-$ $995 \mathrm{~cm}^{-1}$ [7]. Figure 5 shows the results of the FTIR analysis, indicating the increase in the Carbonyl Index, ICO and the Sulfoxide Index, ISO. These first results show that the binder tested shows limited increase of ICO and ISO at lower temperature after RTFOT aging, whereas after PAV aging, similar values were seen regardless of the RTFOT temperature.

Figure 3. DSR results for Bitumen 70/100 at different aging levels. 
Figure 6 shows the complex modulus of one of the binders in virgin state, after RTFOT and PAV aging as well as the recovered binder from the field samples that were in place for four years, indicating that at the same modulus the phase angle has shifted towards smaller values of phase angle indicating loss of the viscous part and increase in the elastic part of the complex modulus. The values of the four year field-aged samples with a void content of about $2.5 \%$ are between those of lab-aged and virgin samples, showing a more severe aging by the laboratory procedure in comparison to the four-year field aged sample. However, it should be noted that the field samples are rather dense and aged mostly in the first few millimetres. In order to make a more proper comparison this top layer only should be investigated.

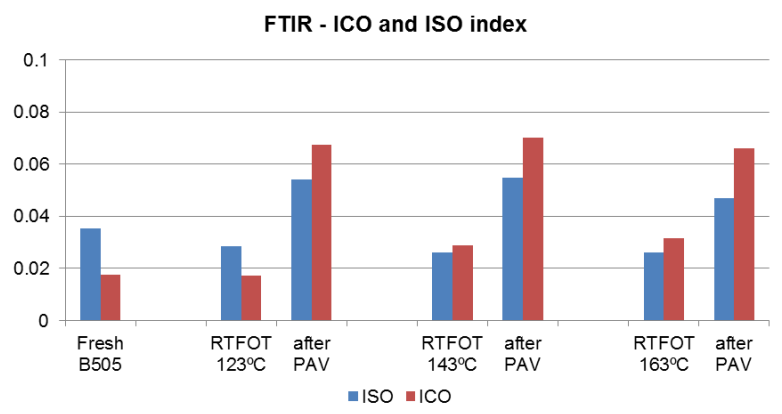

Figure 5. Aging characteristics using FTIR showing the oxidation of the carbonyl and sulfoxide functional groups.

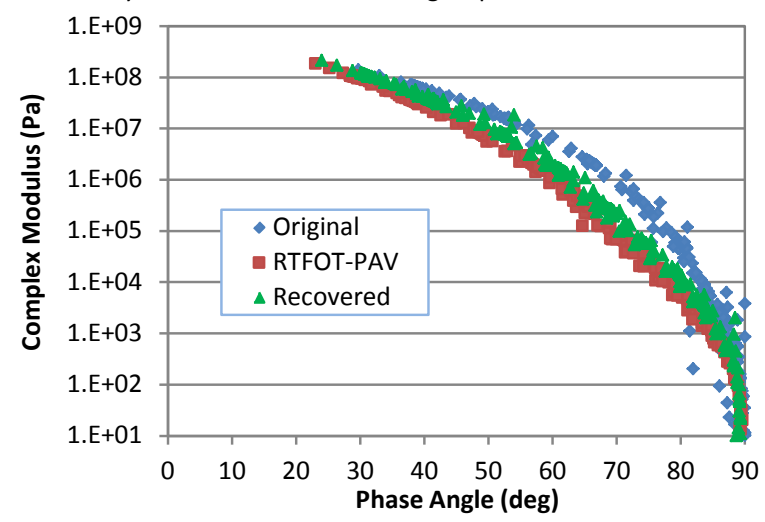

Figure 6. Black diagram showing the complex modulus vs phase angle of original binder laboratory aged (RTFOT+PAV) and recovered field sample.

\section{Preliminary conclusions}

The work has shown that there is a connection between the microstructure, the chemical characteristics and the mechanical characteristics of bituminous binders. Understanding all three aspects allows a holistic understanding of the material behaviour and exploring avenues for its improvement. It was shown that the shortterm aging temperature affects the increase in softening point, while the consequent effect on long-term aging was less pronounced.

\section{Acknowledgements}

The work presented in this article is the result of cooperative efforts in the RILEM TC 252-CMB Chemo-Mechanical Characterization of Bituminous Materials. The authors would like to acknowledge the contribution of all RILEM TC 252CMB members. Q8, Nynas, TU Vienna and Repsol are gratefully thanked for providing the bitumen for the tests.

\section{References}

[1] H.R. Fischer, E.C. Dillingh, C.G.M. Hermse, On the microstructure of bituminous binders. Road Materials and Pavement Design (2014) 15: $1-15$.

[2] H. Soenen et al., Laboratory investigation of bitumen based on round robin DSC and AFM tests. Mater Struct (2014) 47: 1205-1220.

[3] EN 12607-1 Bitumen and bituminous binders. Determination of the resistance to hardening under influence of heat and air. RTFOT method. 2014.

[4] EN 14769:2012 Bitumen and bituminous binders. Accelerated longterm ageing conditioning by a Pressure Ageing Vessel (PAV) 2012.

[5] EN 1426:2007 Bitumen and bituminous binders. Determination of needle penetration. 2007.

[6] EN 1427:2015 Bitumen and bituminous binders - Determination of the softening point - Ring and Ball method. (2015).

[7] P. Marsac et al., Potential and limits of FTIR methods for reclaimed asphalt characterisation. Mater Struct (2014) 47: 1273-1286. 